\title{
In a distinguishing spacetime the horismos relation generates the causal relation
}

\author{
E. Minguzzi *
}

\begin{abstract}
It is proved that in a distinguishing spacetime the horismos relation $E^{+}=$ $J^{+} \backslash I^{+}$generates the causal relation $J^{+}$. In other words two causally related events are joined by a chain of horismotically related events, or again, the causal relation is the smallest transitive relation containing the horismos relation. The result is sharp in the sense that distinction can not be weakened to future or past distinction. Finally, it is proved that a spacetime in which the horismos relation generates the causal relation is necessarily non-total imprisoning.
\end{abstract}

\section{Introduction}

In a spacetime $(M, g)$ (a $C^{r}$ connected, time-oriented Lorentzian manifold, $r \in$ $\{3, \ldots, \infty\}$ of arbitrary dimension $n \geq 2$ and signature $(-,+, \ldots,+))$ we write as usual $p \ll q$ if there is a future directed timelike curve joining $p$ to $q ; p<q$ if there is a future directed causal curve joining $p$ to $q ; p \leq q$ if $p<q$ or $p=q$ and finally 1$] \rightarrow q$ if $p \leq q$ but $p \nless q$. A well known theorem [5, 2] establishes that $p \rightarrow q$ iff either $p=q$ and chronology holds at $p$, or $p$ and $q$ are connected by an achronal lightlike geodesic segment (thus without conjugate points in its interior). The chronological, causal, and horismos relations on $M$ are defined by

$$
\begin{aligned}
I^{+} & =\{(p, q): p \ll q\}, \\
J^{+} & =\{(p, q): p \leq q\}, \\
E^{+} & =\{(p, q): p \rightarrow q\}=J^{+} \backslash I^{+},
\end{aligned}
$$

and of course they are subsets of $M \times M$. While $I^{+}$and $J^{+}$are transitive, the relation $E^{+}$in general is not as the composition of two lightlike achronal geodesics segments may connect chronologically related events.

\footnotetext{
* Dipartimento di Matematica Applicata, Università degli Studi di Firenze, Via S. Marta 3, I-50139 Firenze, Italy. E-mail: ettore.minguzzi@unifi.it

${ }^{1}$ In the definition of $\rightarrow$ or $E^{+}$one can either choose reflexivity, $p \rightarrow p$ for every $p$, as in 8], or to make the equality $E^{+}=J^{+} \backslash I^{+}$always true as in 4 [13. Here we choose the latter possibility, this being equivalent to Kronheimer and Penrose's definition for chronological spacetimes.
} 
The smallest reflexive transitive relation which contains $E^{+}$i: 2

$$
T^{+} \equiv \bigcup_{n=0}^{+\infty}\left(E^{+}\right)^{n}
$$

where it is understood that $\left(E^{+}\right)^{0}=\Delta$ is the diagonal of $M \times M$. We have $(p, q) \in T^{+}$iff $p=q$ or there is a chain of horismotically related events which connects $p$ to $q$. Note that the smallest transitive relation containing $E^{+}$is $\bigcup_{n=1}^{+\infty}\left(E^{+}\right)^{n}$. In a chronological spacetime $E^{+}$is itself reflexive, that is $\Delta \subset E^{+}$, so that the union in Eq. (4) can start from $n=1$, and hence $T^{+}$becomes also the smallest transitive relation containing $E^{+}$. Moreover, in this case $\left(E^{+}\right)^{n} \subset$ $\left(E^{+}\right)^{n+1}$ so that the transitive relation $T^{+}$can also be denoted $E^{+\infty}$ coherently with the notation of 10 .

Since piecewise lightlike geodesics are causal curves we have $T^{+} \subset J^{+}$but the converse inclusion may not hold. In the equality case one can also easily recover the chronological relation as $I^{+}=T^{+} \backslash E^{+}$. This approach in which the relations $I^{+}, J^{+}$and $E^{+}$are recovered from just one of them, in this case $E^{+}$, can be useful to put causality theory in an order theoretic framework in which one focuses on just one relation (usually a partial order).

Under strong causality it is known that $T^{+}=J^{+}$. This result was stated by Kronheimer and Penrose [8, Sect. 2.1] (they state that this equivalence holds if the Alexandrov topology is Hausdorff which is equivalent to strong causality) and a detailed proof can be found for instance in [13, Def. 2.22 and Th. 3.24]. This paper shows that distinction suffices to guarantee the equality $T^{+}=J^{+}$, and in fact that this result is sharp in the sense that distinction can not be weakened to future or past distinction.

Recall that a spacetime is distinguishing if " $I^{+}(x)=I^{+}(y)$ or $I^{-}(x)=$ $I^{-}(y) \Rightarrow x=y$ ". An open neighborhood $U$ distinguishes $p \in U$ if every causal curve $\gamma: I \rightarrow M$, passing through $p$ intersects $U$ only once (i.e. in a connected subset of its domain $I$ ). A spacetime is distinguishing iff every point admits arbitrarily small distinguishing open neighborhood\&3 [5, 13. Analogous definitions in the past and future case exist [13].

The following result holds true

Theorem 1.1. In a past or future distinguishing spacetime the conformal structure is determined by the horismos relation $E^{+}$. In other words if $\left(M, g_{1}\right)$ and $\left(M, g_{2}\right)$ have the same horismos relation, i.e. $E_{1}^{+}=E_{2}^{+}$, then the metrics are conformally related, namely $g_{2}=\Omega g_{1}$, with $\Omega: M \rightarrow(0,+\infty)$.

Proof. Future case. In a future distinguishing spacetime every point admits arbitrarily small future distinguishing open neighborhoods. Given $p \in M$ we can find a neighborhood $U$ which distinguishes $p$ in the future, contained in a convex neighborhood. By future distinction at $p$, we have $J_{U}^{+}(p)=J^{+}(p) \cap U$

\footnotetext{
${ }^{2}$ In the notation of [13] $x \leq{ }^{(\rightarrow)} y$ iff $(x, y) \in T^{+}$.

${ }^{3}$ The proof of this statement contained in [13, Lemma 3.10] is given in the future distinguishing case, the case considered here being analogous.
} 
and $I_{U}^{+}(p)=I^{+}(p) \cap U$, thus $E_{U}^{+}(p)=E^{+}(p) \cap U$. But $E_{U}^{+}(p)$ is the image of exponential map based at $p$, in the spacetime $\left(U,\left.g\right|_{U}\right)$, of the future light cone at $p$. Given $E^{+}(p)$ one determines the light cone at $p$ through the inverse of the exponential map, and thus $E^{+}$fixes the conformal structure of the spacetime (see [16, Appendix D]).

This result is analogous to Malament's theorem [9] which states that in a past or future distinguishing spacetime the causal relation determines the conformal structure (see also [13, Prop 3.13]).

Since in a past or future distinguishing spacetime $E^{+}$fixes the conformal structure, it also clearly determines the causal relation. The non-trivial result established by this work is that in a distinguishing spacetime the causal relation is actually given by $T^{+}$namely by the smallest reflexive transitive relation containing $E^{+}$. Figure 37 of [5] shows an example of past distinguishing spacetime for which $T^{+} \neq J^{+}$, despite the fact that in this spacetime $E^{+}$fixes the conformal structure (the point $p$ in the figure is such that $T^{+}(p)$ equals $E^{+}(p)$ and is given by a portion of the middle lightlike geodesic, thus every point above $p$ although belonging to $I^{+}(p)$ is not in $\left.T^{+}(p)\right)$. This example shows that our result is sharp: distinction can not be weakened to past or future distinction. Nevertheless, distinction is not equivalent to the equality $T^{+}=J^{+}$as the discussion at the end of the next section shows.

\section{The proof and a counterexample to the other direction}

We start with a couple of lemmas.

Lemma 2.1. Assume that $(M, g)$ is distinguishing at $p$, then $p$ admits arbitrarily small globally hyperbolic neighborhoods that distinguish $p$.

Proof. Let $W \ni p$ be an arbitrary small strongly causal neighborhood. There is a neighborhood $V \subset W$ that distinguishes $p$. Let $U \ni p, U \subset V$, be a neighborhood which is both causally convex with respect to $W$ and globally hyperbolic (see [13] remark 2.15). Let $\gamma: I \rightarrow M$ be a causal curve passing through $p$ and let $r \in \gamma \cap U$. It is either $p \leq r$ or $r \leq p$. Let us consider the former case, the latter being analogous. The curve $\gamma$ between $p$ and $r$ is necessarily contained in $V$, as $p, r \in V$, and $V$ distinguishes $p$. Thus it is contained in $W$ and since $U$ is casually convex in $W$, and $p, r \in U$, it is contained in $U$. As $r$ is arbitrary every causal curve passing through $p$ intersects $U$ only once, that is, $U$ distinguishes $p$.

The next lemma is the crucial one in the proof.

Lemma 2.2. Let $V \ni p$ be a globally hyperbolic neighborhood which distinguishes $p$ contained in a convex neighborhood. Then for every $q \in I_{V}^{+}(p)$, the set $E_{V}^{+}(p) \cap$ $E_{V}^{-}(q)$ is non-empty and for every $r \in E_{V}^{+}(p) \cap E_{V}^{-}(q)$ we have $p \rightarrow r \rightarrow q$ where 
$\rightarrow$ stands for the causal relation $E^{+}$in $(M, g)$. Analogously, for every $q \in I_{V}^{-}(p)$, $E_{V}^{+}(q) \cap E_{V}^{-}(p)$ is non-empty and if $r \in E_{V}^{+}(q) \cap E_{V}^{-}(p)$ we have $q \rightarrow r \rightarrow p$.

Proof. Let us prove the former case, the latter being analogous. First $E_{V}^{+}(p) \cap$ $E_{V}^{-}(q)$ is a closed subset of the compact $J_{V}^{+}(p) \cap J_{V}^{-}(q)$ thus it is compact. Let us denote this set with $S$. Since $V$ future distinguishes $p, J_{V}^{+}(p)=J^{+}(p) \cap V$ and $I_{V}^{+}(p)=I^{+}(p) \cap V$ thus $E_{V}^{+}(p)=E^{+}(p) \cap V$. We conclude that for every $r \in S$ it is $p \rightarrow r$. Note that a lightlike geodesic generator of $E_{V}^{+}(p)$ extended towards the future cannot enter $I^{+}(p)$ before escaping $V$, i.e. it cannot enter $I_{V}^{+}(p)$, as $V$ is contained in a convex neighborhood. As a consequence, as long as it stays in $V$ it belongs to $E_{V}^{+}(p)$. In fact it must reach the boundary of the compact set $J_{V}^{+}(p) \cap J_{V}^{-}(q)$, and thus $E_{V}^{-}(q)$, otherwise it would be totally future imprisoned in a compact which is impossible because $V$ is globally hyperbolic. Thus the geodesic generators of $E_{V}^{+}(p)$, once extended to the future intersect $S$, and conversely every point of $S$ is connected to $p$ by a lightlike geodesic. In particular $S$ is non-empty.

Let $r \in S$ and let us prove that $r \rightarrow q$. Assume not, then $r \in S$ can be connected to $q$ with a timelike curve $\gamma$ necessarily not entirely contained in $V$. The causal curve joining $p$ to $r$ and $r$ to $q$ along $\gamma$ is not entirely contained in $V$ and contradicts the fact that $V$ distinguishes $p$.

Theorem 2.3. In a distinguishing spacetime, $J^{+}=T^{+}\left(=E^{+\infty}\right)$.

Proof. $\Rightarrow$. The direction $T^{+} \subset J^{+}$is obvious. Let us prove $J^{+} \subset T^{+}$. Let $x \leq y$, if $y \notin I^{+}(x)$ then $x \rightarrow y$ and there is nothing to prove. Thus assume $x \ll y$, and let $\gamma$ be a timelike curve connecting $x$ to $y$. For every $z \in \gamma$ let $V_{z}$ be a globally hyperbolic neighborhood that distinguishes $z$ contained in a convex neighborhood $W_{z} \supset V_{z}$. Extract a finite subcovering $\left\{V_{z_{n}}\right\}$ from the covering $\left\{V_{z}, z \in \gamma\right\}$ so that $V_{z_{i-1}} \cap V_{z_{i}} \neq \emptyset$. Thus taking $q_{i} \in V_{z_{i-1}} \cap V_{z_{i}}, z_{i-1}$ can be joined to $q_{i}$ with a causal curve made of two achronal lightlike segments, and $q_{i}$ can be joined to $z_{i}$ with a causal curve made of two achronal lightlike segments. Joining all the pieces the searched curve is obtained. In conclusion $(x, y) \in T^{+}$.

It is natural to ask if the equality $T^{+}=J^{+}$implies distinction. The answer is negative. A counterexample is given by the spacetime of figure 2 in 12, although it is not easy to grasp why this spacetime does indeed provide a counterexample. The next theorem establishes some features that any counterexample should have. Recall that a spacetime is non-total imprisoning if no inextendible causal curve is contained in a compact [1, 11. Distinction implies non-total imprisonment which implies causality. Recall also that a lightlike line is an inextendible achronal causal curve and thus a lightlike geodesic.

Theorem 2.4. If a spacetime $(M, g)$ is such that $T^{+}=J^{+}$then it is non-total imprisoning. Moreover, either (a) $(M, g)$ is future distinguishing or (b) there is a lightlike line $\sigma$ and a last point (in the future direction) $w \in \sigma$ with the property that $\sigma \subset \overline{I^{+}(w)}$. A past version also holds. 
Proof. Let us assume that $T^{+}=J^{+}$. The spacetime is chronological otherwise there would be a point $p$ inside the chronology violating set. Since the chronology violating class $I^{+}(p) \cap I^{-}(p)$ is open and contains $p$ no achronal lightlike geodesic segment can start from $p$, thus $J^{+}(p)=T^{+}(p)=\{p\}$, which is a contradiction.

The spacetime is non-total imprisoning, indeed otherwise there would be a totally imprisoned causal curve, and because of chronology there would be an achronal (minimal invariant) set $\Omega$ generated by lighlike lines such that all the points in the set have the same chronological past and future [11, Theorem 3.9]. Let $x \in \Omega$, since through $x$ there passes a lightlike line $\gamma$ included in $\Omega$, we can take $y \in \gamma$ before $x$. Any point $z \in J^{+}(x)$ which does not belong to $\gamma$ belongs to $I^{+}(y)$, because if $x$ is connected to $z$ by a lightlike geodesic segment then it does not join smoothly with $\gamma$ at $x$ as $z \notin \gamma$. But $I^{+}(y)=I^{+}(x)$ thus $E^{+}(x) \subset \gamma$. Since the same argument holds for every point in $\gamma, T^{+}(x) \subset \gamma$, but $I^{+}(x)$ contains points which are not in $\gamma$ because $\Omega$ is achronal, a contradiction.

Let us assume that the spacetime is not future distinguishing, then we can find a point $x \in M$ and a sequence of causal curve $\sigma_{n}$ of starting point $x$ and ending point $z_{n}$ with $z_{n} \rightarrow x$, and a neighborhood $V \ni x$ such that none of the $\sigma_{n}$ is contained in $V$. By causality an application of the limit curve theorem gives us a future inextendible limit continuous causal curve $\sigma^{x}$ starting from $x$ and a past inextendible limit continuous causal curve $\sigma^{z}$ ending at $x$. The causal curve $\sigma=\sigma^{x} \circ \sigma^{z}$ is inextendible and achronal, thus a lightlike line, otherwise chronology would be violated. Note that since all the points of $\sigma$ are limit points of a subsequence of $\sigma_{n}, \sigma \subset \overline{I^{+}(x)}$. Note that if a point $y \in \sigma$ has the property $\sigma \subset \overline{I^{+}(y)}$ then the same is true for all the points which come before $y$ on $\sigma$. Moreover, if a point $y$ has this property then it has the same chronological future of the points which come before it on $\sigma$. As a consequence for a point $y$ sharing this property $E^{+}(y) \subset \sigma$, indeed a point $z \in E^{+}(y) \backslash \sigma$ would be chronologically related with the points before $y$ (the lightlike geodesics do not join smoothly at $y$ ) and hence with $y$, a contradiction. Thus not all the points on $\sigma$ can share the mentioned property otherwise $T^{+}(y) \subset \sigma$ while $I^{+}(y), I^{+}(y) \subset T^{+}(y)$, has clearly points which do not belong to $\sigma$. Thus there is a point $w \in \sigma$ such that all the points $y \in \sigma$ before it share the mentioned property while those after it do not. We have to show that $w$ shares the property. Since for every $y \in \sigma$ before $w$, we have $E^{+}(y) \subset \gamma$ we also have $T^{+}(y) \subset J^{+}(w)$ because the chain of $E^{+}$related events starting from $y$ can leave $\sigma$ only after having left the portion of $\sigma$ before $w$. Thus $I^{+}(y) \subset T^{+}(y) \subset J^{+}(w)$ and hence $y \in \overline{J^{+}(w)}$. As it holds for every $y$ before $w$ and it clearly holds for every points after $w$ we have $\sigma \subset \overline{I^{+}(w)}$.

\section{Conclusions}

It has been proved that in a distinguishing spacetime the horismos relation generates the causal relation, namely $E^{+\infty}=J^{+}$, and that the result is optimal 
in the sense that distinction can not be weakened to future or past distinction.

As previously mentioned this result may be useful in all those approaches which try to replace the spacetime with a partially ordered set. This is the route taken for instance in the Causal Set Theory of quantum gravity [3, 15, 6]. Here an inspiring result is the already mentioned Malament's theorem [9] which states that in a distinguishing spacetime the causal relation determines the conformal structure of spacetime. The conformal factor can be fixed through the notion of volume on spacetime. If one picks up points from the spacetime in a random way so that equal volumes have equal average number of picked up points, and connects them through the causal relation one obtains a causal set, that is, a discrete version of the original spacetime. The idea is that one can start from an abstract notion of causal set, regard it as fundamental, and study in which limit it approximates the usual spacetime continuum.

If the distinction property suitably extended to the discrete domain holds then we can have the reasonable hope to get the right continuum limit as the abstract causal relation imbedded in the causal set would pass to a continuum causal relation which in turn determines the light cone structure. It is interesting to note that at least the property of non-total imprisonment is always satisfied in a causal set, indeed the local finiteness property [3, 15, 6] implies that every causal curve entering a finite volume would pass through the finite number of vertices there contained to finally escape.

The result proved in this work may be useful to build up an abstract framework in which the horismos rather that the causal relation, plays the fundamental role. This possibility is already suggested by causal set theory in which there seems to be a somewhat unexplored analogy (but see [14]) between the concept of horismos relation on the continuum side and that of link [7] on the causal set (discrete) side. Both of them, in the respective contexts, generate the causal relation.

\section{Acknowledgments}

This work has been partially supported by GNFM of INDAM. My interest on a possible improvement of Kronheimer and Penrose's result arose while writing the review [13] jointly with M. Sánchez. Useful conversations with him on this problem are acknowledged.

\section{References}

[1] J. K. Beem. Conformal changes and geodesic completeness. Commun. Math. Phys., 49:179-186, 1976.

[2] J. K. Beem, P. E. Ehrlich, and K. L. Easley. Global Lorentzian Geometry. Marcel Dekker Inc., New York, 1996. 
[3] L. Bombelli, J.-H. Lee, D. Meyer, and R. D. Sorkin. Space-time as a causal set. Phys. Rev. Lett., 59:521-524, 1987.

[4] A. García-Parrado and J. M. M. Senovilla. Causal structures and causal boundaries. Class. Quantum Grav., 22:R1-R84, 2005.

[5] S. W. Hawking and G. F. R. Ellis. The Large Scale Structure of Space-Time. Cambridge University Press, Cambridge, 1973.

[6] J. Henson. The causal set approach to quantum gravity. To be published in 'Approaches to Quantum Gravity - Towards a new understanding of space and time' (ed. D. Oriti), Cambridge University Press. arXiv:gr-qc/0601121v2, 2006.

[7] S. Johnston. Particle propagators on discrete spacetime. Class. Quantum Grav., 25:202001, 2008.

[8] E. H. Kronheimer and R. Penrose. On the structure of causal spaces. Proc. Camb. Phil. Soc., 63:482-501, 1967.

[9] D. B. Malament. The class of continuous timelike curves determines the topology of spacetime. J. Math. Phys., 18:1399-1404, 1977.

[10] E. Minguzzi. The causal ladder and the strength of $K$-causality. I. Class. Quantum Grav., 25:015009, 2008.

[11] E. Minguzzi. Non-imprisonment conditions on spacetime. J. Math. Phys., 49:062503, 2008

[12] E. Minguzzi. Weak distinction and the optimal definition of causal continuity. Class. Quantum Grav., 25:075015, 2008.

[13] E. Minguzzi and M. Sánchez. The causal hierarchy of spacetimes, volume H. Baum, D. Alekseevsky (eds.), Recent developments in pseudo-Riemannian geometry, of ESI Lect. Math. Phys., pages 299-358. Eur. Math. Soc. Publ. House, Zurich, 2008. arXiv:gr-qc/0609119.

[14] L. Philpott, F. Dowker, and R. Sorkin. Energy-momentum diffusion from spacetime discreteness. arXiv:0810.5591, 2008.

[15] R. D. Sorkin. First steps with causal sets. In R. Cianci et al., editor, Proceedings of the ninth Italian conference in General Relativity and Gravitational Physics, pages 68-90, Singapore, 1991. World Scientific.

[16] R. M. Wald. General Relativity. The University of Chicago Press, Chicago, 1984. 